\title{
Outcomes of Left Atrial Ablation in Patients with Prior Mitral Valve Surgery
}

\author{
Shu Chang ${ }^{1}$, Heidi Huber ${ }^{2}$, Matthew Zipse ${ }^{1}$, Alexis Tumolo ${ }^{1}$, Michael Rosenberg${ }^{1}$, Jose \\ Sanchez $^{3}$, Ryan Borne ${ }^{1}$, John West ${ }^{1}$, Amneet Sandhu ${ }^{1}$, Paul Varosy ${ }^{1}$, Wendy Tzou ${ }^{2}$, and \\ Ryan Aleong ${ }^{1}$ \\ ${ }^{1}$ University of Colorado Denver School of Medicine \\ ${ }^{2}$ University of Colorado \\ ${ }^{3}$ University of Colorado Denver - Anschutz Medical Campus
}

November 4, 2020

\begin{abstract}
Background: Patients with a history of mitral valve (MV) surgery can present with unique challenges during left atrial (LA) ablation due to abnormal atrial substrate and descriptions of ablation in this cohort is limited. We aim to evaluate LA ablation characteristics and outcomes in patients with a history of mitral valve surgery. Objective: We hypothesize that the success rate for ablations of LA arrhythmias in patients with prior MV surgery will be inferior to patients without prior MV surgery due to left atriopathy, presence of a MV prosthesis, and a higher burden of pre-ablation LA scar. Methods: In this single center, retrospective study, we evaluated patients who had a history of MV surgery and underwent LA ablation between January 2013 and May 2019. We analyzed baseline patient characteristics, type of MV disease and surgery, available pre-ablation voltage maps, and ablation outcomes. Results: We present a series of 20 patients who underwent a total of 30 LA ablation procedures. All 20 patients underwent pulmonary vein isolation and 11 patients also underwent ablation for LA macro-reentrant flutters. The majority of the patients $(55 \%)$ were without recurrent documented arrhythmias at a mean follow-up of 22 months postablation. Two patients had acutely unsuccessful ablation. Conclusions: Although LA ablation in patients with previous MV surgery can be challenging due to abnormal atrial substrate and the presence of the valve prosthesis, the majority of patients in our cohort experienced atrial arrhythmia free survival at a mean follow-up of 22 months.
\end{abstract}

\section{Outcomes of Left Atrial Ablation in Patients with Prior Mitral Valve Surgery}

Short title: Ablation Outcomes in Patients with Mitral Surgery

Shu C. Chang, MD ${ }^{\mathrm{a}}$; Heidi Huber, RNª; Matthew Zipse, MD ${ }^{\mathrm{a}}$; Alexis Tumolo, MD ${ }^{\mathrm{a}}$; Michael Rosenberg, $\mathrm{MD}^{\mathrm{a}}$; Jose Sanchez, $\mathrm{MD}^{\mathrm{a}}$; Ryan Borne, MD ${ }^{\mathrm{a}}$; J. Jason West, $\mathrm{MD}^{\mathrm{a}}$; Amneet Sandhu, MD ${ }^{\mathrm{a}}$; Paul Varosy, $\mathrm{MD}^{\mathrm{a}}$; Wendy Tzou, MD, FHRS ${ }^{\mathrm{a}}$; Ryan G.Aleong, $\mathrm{MD}^{\mathrm{a}}$

Affiliation: ${ }^{a}$ University of Colorado Anschutz Medical Center - 12401 East 17th Avenue, B136

Aurora, CO 80045

\section{Corresponding Author:}

Ryan G. Aleong, MD

Section of Cardiac Electrophysiology

University of Colorado Hospital

12401 East 17th Avenue, B136 
Aurora, CO 80045

Telephone: (720) 848-6558

Fax: (720) 848-7314

E-mail: ryan.aleong@cuanschutz.edu

Conflicts of Interest: No conflicts of interest to disclose

Funding: This research did not receive any specific grant from funding agencies in the public, commercial, or not-for-profit sectors.

Word Count: 3,586

\section{Abstract \\ Background:}

Patients with a history of mitral valve (MV) surgery can present with unique challenges during left atrial (LA) ablation due to abnormal atrial substrate and descriptions of ablation in this cohort is limited. We aim to evaluate LA ablation characteristics and outcomes in patients with a history of mitral valve surgery.

\section{Objective:}

We hypothesize that the success rate for ablations of LA arrhythmias in patients with prior MV surgery will be inferior to patients without prior MV surgery due to left atriopathy, presence of a MV prosthesis, and a higher burden of pre-ablation LA scar.

\section{Methods:}

In this single center, retrospective study, we evaluated patients who had a history of MV surgery and underwent LA ablation between January 2013 and May 2019. We analyzed baseline patient characteristics, type of MV disease and surgery, available pre-ablation voltage maps, and ablation outcomes.

\section{Results:}

We present a series of 20 patients who underwent a total of $30 \mathrm{LA}$ ablation procedures. All 20 patients underwent pulmonary vein isolation and 11 patients also underwent ablation for LA macro-reentrant flutters. The majority of the patients (55\%) were without recurrent documented arrhythmias at a mean follow-up of 22 months post-ablation. Two patients had acutely unsuccessful ablation.

\section{Conclusions:}

Although LA ablation in patients with previous MV surgery can be challenging due to abnormal atrial substrate and the presence of the valve prosthesis, the majority of patients in our cohort experienced atrial arrhythmia free survival at a mean follow-up of 22 months.

\section{Abbreviation List:}

LA - Left atrial

MV - Mitral valve

PVI - Pulmonary vein isolation

MA - Mitral annular

\section{Key Words:}

Left atrial arrhythmia, left atrial scar, catheter ablation, mitral valve surgery, mitral valve disease, macroreentrant arrhythmia

\section{Introduction:}


Catheter ablation has become an increasingly utilized treatment strategy for left atrial (LA) arrhythmias for patients with complex cardiac disease. Recently published literature report a significant reduction in mean atrial fibrillation (AF) burden following ablation for atrial arrhythmias with potential mortality benefits in select patient populations. ${ }^{1,2}$ This has been accomplished with an overall combined ablation related major complication rate of death, pericardial effusion, phrenic nerve injury, pulmonary vein stenosis, and atrial-esophageal fistula at $<5 \% .{ }^{1-3}$

LA catheter ablation in patients with a history of severe mitral valve (MV) disease and previous mitral valve surgery carries additional complexities and challenges compared to patients without a history of MV disease. Patients with mitral valve disease have a propensity to develop LA remodeling and scar formation. ${ }^{4,}{ }^{5}$ In addition, patients that undergo surgical MV intervention may develop additional LA substrate from surgical scar. ${ }^{6,7}$ There are few reports detailing the safety and efficacy of catheter ablation for atrial arrhythmias arising from the left atrium in this cohort. ${ }^{7-9}$

Mountantonakis et al. showed that catheter ablation for mitral annular flutter in patients with a history of mitral valve surgery is feasible with acceptable rates of complications in a cohort of 21 patients. ${ }^{8}$ In addition, Enriquez et al. demonstrated that right and LA tachycardias arising from varied locations can be mapped and ablated with reasonable outcomes in this setting. ${ }^{7}$ Lang et al. and Bai et al. also demonstrated that comparable success rates can be achieved for AF ablation procedures in patients with MV prosthesis compared to patients without. ${ }^{9}{ }^{10}$ In our study, we examine ablation outcomes for all LA arrhythmias in patients with a history of mitral valve surgery. In addition, we evaluate pre-ablation LA sinus voltage maps in order to assess for any correlation between baseline scar burden and ablation outcomes. To the best of our knowledge, there has not been a published report focusing on solely LA ablation outcomes in patients with prior MV surgery. Furthermore, to the best of our knowledge, this is the first assessment of pre-ablation LA scar burden in relation to success of LA arrhythmia ablation in patients with a history of mitral valve surgery. We hypothesize that the success rate for ablations of LA arrhythmias in patients with prior MV surgery will be inferior to patients without prior MV surgery due to left atriopathy, presence of a MV prosthesis, and a higher burden of pre-ablation LA scar.

\section{Methods:}

In this single center, retrospective study, we evaluated patients that presented to the electrophysiology laboratory at the University of Colorado Anschutz Medical Center. Patients were included in this study if they were greater than 18 years of age, had a history of MV surgery and underwent a LA ablation procedure between January 2013 and May 2019. Baseline characteristics of each patient was analyzed including age, gender, type of MV disease and surgery, LV systolic function, type of documented arrhythmia, number of ablations, and type of ablations performed. Three-dimension electroanatomic maps were generated using the CARTO\& advanced 3D mapping system (Biosense Webster, Diamond Bar, CA, USA). In patients that possessed a pre-ablation LA high definition sinus rhythm voltage map, we assessed total endocardial surface area, endocardial surface area of scar, and percent scar burden. Endocardial scar was defined as a bipolar voltage of less than or equal to $0.1 \mathrm{mV}$. Ablation outcome analysis was performed including acute procedural success, complications, presence and time to arrhythmia occurrence, and duration of freedom from recurrent symptomatic arrhythmias.

\section{Statistical Methods}

Variables were analyzed using means, standard deviations, median values, and proportions where appropriate. Categorical variables were analyzed with ?2 tests or Fisher's exact test. Continuous variables were compared using Student's t-test. P values were significant at P [?]0.05 and were two sided. The study was approved by the institutional review board at the University of Colorado Anschutz Medical Center.

\section{Results:}

\section{Ablation Outcomes}

Between January 2013 and May 2019, a total of 20 patients with a history of MV surgery underwent LA 
ablation for an atrial arrhythmia at the University of Colorado Anschutz Medical Center. The mean age of the patients was 62 years with a range from 22 to 79 years. The majority of patients (80\%) had preserved LV systolic function. Severe non-rheumatic mitral valve regurgitation was the most frequent indication for MV surgery (13 patients) followed by severe rheumatic mitral valve disease ( 5 patients). Two patients had a history of MV surgery for congenital dysplastic mitral valve disease. Nine of the 20 patients received a mechanical MV replacement, 7 received a bioprosthetic MV replacement, and 4 patients received MV repair. Eighteen of the 20 patients had documented paroxysmal or persistent atrial fibrillation and 11 of the 20 patients had documented atypical flutter (Table 1).

A total of 30 left atrial ablation procedures were performed on the 20 patients in this cohort from January 2013 to May 2019. Six of the 20 patients underwent more than 1 ablation procedure and 1 of the patients underwent $4 \mathrm{LA}$ ablation procedures in that time frame. All 20 patients underwent pulmonary vein isolation. Eleven patients received additional LA ablation lesions beyond standard pulmonary vein isolation. These included 10 who received a MA ablation line, 7 who received ablation for other non-mitral annular macroreentrant LA flutters, and 3 patients who received additional ablation for a focal left atrial tachycardia. Acute success, defined as inability to induce atrial fibrillation or atrial tachycardia at the end of the procedure, was achieved in 18 of the 20 patients (Table 2). In one patient, MA flutter was refractory to ablation because of the inability to generate adequate ablation lesions at the mechanical valve annulus due to impedance spikes. In the second acutely unsuccessful patient, the ablation procedure had to be terminated abruptly due to compromise of their mechanical MV prothesis which involved a catheter being entrapped in the valve apparatus.

In long-term follow up, 11 of the 20 patients were free from documented symptomatic arrhythmia recurrence. The mean arrhythmia free duration was 21.7 months with a range of 6 to 39 months (Table 2). In the 9 patients with arrhythmia recurrence, 5 patients experienced recurrence within 3 months of their last ablation and all patients recurred within 1 year. In regard to type of MV surgery performed, four of the nine patients with a mechanical mitral valve replacement were free from documented symptomatic arrhythmia recurrence compared to five of the seven patients with a bioprosthetic mitral valve replacement and two of the four patients that underwent mitral valve repair. Six of the patients has concomitant surgical ablation in the left atrium during MV surgery. Of these six patients, two patients were free from documented symptomatic arrhythmia recurrence.

\section{Sinus Rhythm Voltage Map Analysis}

In 13 of the 20 patients, a detailed pre-ablation sinus rhythm voltage map was obtained. In the remaining 7 patients, a pre-ablation voltage map was not available for analysis either because the patient presented in an atrial tachycardia or a detailed LA voltage map was not performed prior to start of ablation. The baseline demographics of these patients are detailed in Table 3. All sinus rhythm bipolar voltage points were obtained prior to any endocardial catheter ablation and were obtained using a high-resolution mapping catheter (Figure 1). An average of 1881 points were obtained per patient with a standard deviation of 1293 points. The mean left atrial endocardial surface area was $124 \mathrm{~cm}^{2}$ with a standard deviation of $46 \mathrm{~cm}^{2}$. The mean endocardial scar (defined as bipolar voltage of less than or equal to $0.1 \mathrm{mV}$ ) area was $44 \mathrm{~cm}^{2}$, which equated to a mean $29.3 \%$ scar burden with a standard deviation of $23.6 \%$ (Table 4 ).

In 8 of the 13 patients, the endocardial scar burden was less than the mean. The mean scar burden of these 8 patients was $12.7 \%$. Of these 8 patients, $5(63 \%)$ were free from recurrent arrhythmia after their index ablation procedure with a mean follow up time of 20 months. The mean scar burden of the 5 patients above the mean was $55 \%$. Four of these 5 patients with a high burden of endocardial scar experienced recurrence of arrhythmia within 3 months including 1 patient who experienced acutely unsuccessful ablation attempt (Figure 2).

\section{Discussion:}

We present a retrospective series of 20 patients with a history of mitral valve surgery that underwent LA catheter ablation for LA arrhythmias. Our analysis shows that LA ablation in patients with previous mitral 
valve surgeries can be performed with reasonable efficacy and relatively low complication rates. Overall, we showed that $55 \%$ of our patients were free of documented symptomatic arrhythmias for a mean of almost 22 months. These findings add to earlier investigations by Mountantonakis et al. and Enriquez et al. which showed that mapping and ablating left and right atrial arrhythmias in patients with a history of MV surgery is feasible. ${ }^{7,8}$

In addition, we were able to analyze pre-ablation sinus rhythm voltage maps in a subset of our cohort. To the best of our knowledge, there are no universally accepted definitions regarding what value of atrial bipolar voltage value best correlates with scar and how to quantify the burden of scar using a 3-dimensional electroanatomic mapping software. ${ }^{5,6}$ Thirteen of our 20 patients had a pre-ablation voltage map available for analysis. There was significant heterogeneity in the abundance of LA scar in our 13 patients with a pre-ablation sinus rhythm voltage map. The 8 patients that possessed relatively less LA scar burden had a better long-term arrhythmia free survival rate $(63 \%)$ versus the patients with relatively more baseline scar burden (20\%). It is unclear whether burden of left atrial scar should impact ablation approach however, it may be used as a predictor of arrhythmia recurrence.

Patients with atriopathy from MV disease have a high propensity to develop LA arrhythmias. Prior studies have shown that patients with MV disease have a 30-50\% prevalence of atrial fibrillation. ${ }^{11}$ In addition, the prevalence of LA arrhythmias appears to increase after MV surgery. ${ }^{12}$ Lukac et al showed that patients with prior MV surgery have an estimated $20-45 \%$ prevalence of atrial tachycardias and a greater than $50 \%$ prevalence of atrial fibrillation following MV surgery. ${ }^{12}$ Prior studies have shown mixed results from concomitant surgical ablation at the time of mitral valve surgery in relation to prevalence of LA arrhythmias. Gillinov et al found that concomitant surgical ablation during MV surgery improved the prevalence of atrial fibrillation during a 6-12 month follow up (36\% in patient who received surgical ablation vs $70 \%$ in those who did not). ${ }^{11}$ However other studies have shown that the addition of surgical ablation at the time of MV surgery can predispose patients to more LA arrhythmias. ${ }^{7,} 13$ Thus, continued investigation of the arrhythmogenic properties of LA scar is important in understanding the etiology of LA arrhythmias in patients with prior MV surgery.

Despite the high burden of LA arrhythmias in patients with prior MV surgery, many providers remain hesitant to perform LA catheter ablation in this cohort due to potential complications involving the MV protheses. Prior studies have demonstrated feasibility and safety of catheter-based radiofrequency ablation of LA arrhythmias in patients with history of MV surgery, however these studies were performed in high volume centers with experienced operators. ${ }^{7,} 8,14$ Our study also demonstrates that reasonable outcomes can be attained with catheter ablation of LA arrhythmias in patients with prior MV surgery. In our study, although the overall major complication rate was low, we did experience one major complication involving the entrapment of a high-density mapping catheter in a mechanical valve prosthesis. Given the potential benefits of catheter ablation for LA arrhythmias in patients with prior MV surgery, careful planning and appropriate selection of catheters is imperative to minimize unique complications associated with this patient population.

\section{Limitations}

The major limitation to this study is the small cohort of patients presented. Overall, a small fraction of patients undergoing catheter ablation in an electrophysiology lab will meet the inclusion criteria used in this study. Furthermore, an even smaller number of patients will have a pre-ablation sinus rhythm voltage map obtained during ablation of left atrial arrhythmias. A larger cohort, potentially multicenter would allow us to make a more thorough analysis of ablation outcomes in this specific cohort. Another limitation is the standardization of pre-ablation left atrial voltage maps. We selected pre-ablation left atrial voltage maps for analysis if they contained a high density of bipolar voltage points in sinus rhythm. We assumed that maps with a high density of points generated a good representation of the total LA endocardial surface area.

\section{Future Directions}

Further study is needed in this cohort of patients. Currently only small single-center studies have been 
published due to the complexity of this patient population. A multicenter analysis could provide better estimations of success and complication rates that can be discussed with patients when deciding whether to proceed with ablation. In addition, further pre-ablation scar analysis can help determine whether preablation scar burden predicts likelihood of arrhythmia recurrence. An important consideration in this cohort is complications related to the mitral valve prosthesis. We report on 1 complication experienced in our cohort however no complications were reported in prior studies cited in this paper. A more thorough assessment of potential MV prosthesis complications through a larger multicenter study may help providers better discuss the risks of the procedure with patients.

\section{Conclusions:}

Although left atrial ablation in patients with previous mitral valve surgery can be challenging due to abnormal atrial substrate and the valve prosthesis, the majority of patients in our cohort experienced atrial arrhythmia free survival at a mean follow up of 22 months. One patient experienced a major complication involving their mechanical MV prosthesis. In addition, higher baseline pre-ablation endocardial scar burden defined as bipolar voltage of $<0.1 \mathrm{mV}$ using a 3-dimensional electroanatomic mapping software led to inferior outcomes.

\section{References:}

1. Kuck KH, Brugada J, Furnkranz A, Metzner A, Ouyang F, Chun KR, Elvan A, Arentz T, Bestehorn K, Pocock SJ, Albenque JP, Tondo C, Fire and Investigators ICE. Cryoballoon or Radiofrequency Ablation for Paroxysmal Atrial Fibrillation. N Engl J Med . 2016;374:2235-45.

2. Marrouche NF, Brachmann J, Andresen D, Siebels J, Boersma L, Jordaens L, Merkely B, Pokushalov E, Sanders P, Proff J, Schunkert H, Christ H, Vogt J, Bansch D and Investigators C-A. Catheter Ablation for Atrial Fibrillation with Heart Failure. N Engl J Med . 2018;378:417-427.

3. Deshmukh A, Patel NJ, Pant S, Shah N, Chothani A, Mehta K, Grover P, Singh V, Vallurupalli S, Savani GT, Badheka A, Tuliani T, Dabhadkar K, Dibu G, Reddy YM, Sewani A, Kowalski M, Mitrani R, Paydak $\mathrm{H}$ and Viles-Gonzalez JF. In-hospital complications associated with catheter ablation of atrial fibrillation in the United States between 2000 and 2010: analysis of 93801 procedures. Circulation . 2013;128:2104-12.

4. Foglieni C, Rusconi R, Mantione ME, Fragasso G, Alfieri O and Maisano F. Early left atrial tissue features in patients with chronic mitral regurgitation and sinus rhythm: Alterations of not remodeled left atria.International journal of cardiology . 2016;219:433-8.

5. Schaeffer B, Akbulak RO, Jularic M, Moser J, Eickholt C, Schwarzl JM, Klatt N, Kuklik P, Meyer C and Willems S. High-Density Mapping and Ablation of Primary Nonfocal Left Atrial Tachycardia: Characterizing a Distinct Arrhythmogenic Substrate. JACC Clin Electrophysiol . 2019;5:417-426.

6. Markowitz SM, Brodman RF, Stein KM, Mittal S, Slotwiner DJ, Iwai S, Das MK and Lerman BB. Lesional tachycardias related to mitral valve surgery. J Am Coll Cardiol . 2002;39:1973-1983.

7. Enriquez A, Santangeli P, Zado ES, Liang J, Castro S, Garcia FC, Schaller RD, Supple GE, Frankel DS, Callans DJ, Lin D, Dixit S, Deo R, Riley MP and Marchlinski FE. Postoperative atrial tachycardias after mitral valve surgery: Mechanisms and outcomes of catheter ablation. Heart Rhythm . 2017;14:520-526.

8. Mountantonakis S, Frankel DS, Hutchinson MD, Dixit S, Riley M, Callans DJ, Garcia F, Lin D, Tzou W, Bala R, Marchlinski FE and Gerstenfeld EP. Feasibility of catheter ablation of mitral annular flutter in patients with prior mitral valve surgery. Heart Rhythm . 2011;8:809-14.

9. Bai R, L DIB, Mohanty P, Santangeli P, Mohanty S, Pump A, Elayi CS, Reddy YM, Forleo GB, Hongo R, Beheiry S, Russo AD, Casella M, Pelargonio G, Santarelli P, Horton R, Sanchez J, Gallinghouse J, Burkhardt JD, Ma C, Lakkireddy D, Tondo C and Natale A. Catheter ablation of atrial fibrillation in patients with mechanical mitral valve: long-term outcome of single procedure of pulmonary vein antrum isolation with or without nonpulmonary vein trigger ablation. J Cardiovasc Electrophysiol . 2014;25:824-833. 
10. Lang CC, Santinelli V, Augello G, Ferro A, Gugliotta F, Gulletta S, Vicedomini G, Mesas C, Paglino G, Sala S, Sora N, Mazzone P, Manguso F and Pappone C. Transcatheter radiofrequency ablation of atrial fibrillation in patients with mitral valve prostheses and enlarged atria: safety, feasibility, and efficacy. $J A m$ Coll Cardiol . 2005;45:868-72.

11. Gillinov AM, Gelijns AC, Parides MK, DeRose JJ, Jr., Moskowitz AJ, Voisine P, Ailawadi G, Bouchard D, Smith PK, Mack MJ, Acker MA, Mullen JC, Rose EA, Chang HL, Puskas JD, Couderc JP, Gardner TJ, Varghese R, Horvath KA, Bolling SF, Michler RE, Geller NL, Ascheim DD, Miller MA, Bagiella E, Moquete EG, Williams P, Taddei-Peters WC, O'Gara PT, Blackstone EH, Argenziano M and Investigators C. Surgical ablation of atrial fibrillation during mitral-valve surgery. N Engl J Med . 2015;372:1399-409.

12. Lukac P, Hjortdal VE, Pedersen AK, Mortensen PT, Jensen HK and Hansen PS. Atrial incision affects the incidence of atrial tachycardia after mitral valve surgery. Ann Thorac Surg . 2006;81:509-13.

13. Wazni OM, Saliba W, Fahmy T, Lakkireddy D, Thal S, Kanj M, Martin DO, Burkhardt JD, Schweikert $\mathrm{R}$ and Natale A. Atrial arrhythmias after surgical maze: findings during catheter ablation. $J$ Am Coll Cardiol . 2006;48:1405-9.

14. Hussein AA, Wazni OM, Harb S, Joseph L, Chamsi-Pasha M, Bhargava M, Martin DO, Dresing T, Callahan T, Kanj M, Natale A, Lindsay BD and Saliba WI. Radiofrequency ablation of atrial fibrillation in patients with mechanical mitral valve prostheses safety, feasibility, electrophysiologic findings, and outcomes. J Am Coll Cardiol . 2011;58:596-602.

Table 1: Baseline demographics of cohort of 20 patients 


\begin{tabular}{|c|c|}
\hline Baseline Demographics & $N=20$ \\
\hline Male Gender & $8(40 \%)$ \\
\hline Mean Age (Range) in Years & $62(22-79)$ \\
\hline \multicolumn{2}{|l|}{ Ejection Fraction } \\
\hline$>52 \%$ & $16(80 \%)$ \\
\hline $40-52 \%$ & $2(10 \%)$ \\
\hline$<40 \%$ & $2(10 \%)$ \\
\hline \multicolumn{2}{|l|}{ Indication for MV surgery } \\
\hline Severe Regurgitation & $13(65 \%)$ \\
\hline Rheumatic Valve Disease & $5(25 \%)$ \\
\hline Dysplastic Mitral Valve & $2(10 \%)$ \\
\hline \multicolumn{2}{|l|}{ Type of MV Surgery } \\
\hline Mechanical MV Replacement & $9(45 \%)$ \\
\hline Bioprosthetic MV Replacement & $7(35 \%)$ \\
\hline MV Repair & $4(20 \%)$ \\
\hline History of Surgical Ablation & $6(30 \%)$ \\
\hline \multicolumn{2}{|l|}{ Type of Arrhythmia } \\
\hline Paroxysmal AF & $7(35 \%)$ \\
\hline Persistent AF & $11(55 \%)$ \\
\hline MA Flutter & $10(50 \%)$ \\
\hline Focal LAAT & $2(10 \%)$ \\
\hline Patients With >1 Arrhythmia & $9(45 \%)$ \\
\hline
\end{tabular}

Table 2: Ablation data for cohort of 20 patients 


\begin{tabular}{|c|c|}
\hline Ablation Data & \\
\hline Total Number of Ablation Procedures & 30 \\
\hline Patients With >1 Ablation & $6(30 \%)$ \\
\hline \multicolumn{2}{|l|}{ Type of Ablation } \\
\hline PVI & $20(100 \%)$ \\
\hline MA Flutter Line & $10(50 \%)$ \\
\hline Other LA Flutter Lines & $7(35 \%)$ \\
\hline Focal LAAT & $3(15 \%)$ \\
\hline Patients Free of Arrhythmia at Follow-up & $11(55 \%)$ \\
\hline $\begin{array}{l}\text { Continued Antiarrhythmic Therapy in Patients Free of } \\
\text { Arrhythmia }\end{array}$ & $4(36 \%)$ \\
\hline $\begin{array}{l}\text { Mean Arrhythmia Free Duration (Range) } \\
\text { in Months }\end{array}$ & $21.7(6-39)$ \\
\hline Patients With Severe Complications & $1(5 \%)$ \\
\hline
\end{tabular}

Table 3: Baseline demographics of subset of 13 patients with available pre-ablation sinus rhythm voltage map

\begin{tabular}{|c|c|}
\hline Baseline Demographics & $N=13$ \\
\hline Male Gender & $5(38 \%)$ \\
\hline Mean Age (Range) in Years & $59(22-75)$ \\
\hline \multicolumn{2}{|l|}{ Ejection Fraction } \\
\hline$>52 \%$ & $10(77 \%)$ \\
\hline $40-52 \%$ & $2(15 \%)$ \\
\hline$<40 \%$ & $1(8 \%)$ \\
\hline \multicolumn{2}{|l|}{ Type of MV Disease } \\
\hline Severe Regurgitation & $9(69 \%)$ \\
\hline Rheumatic Valve Disease & $2(15 \%)$ \\
\hline Dysplastic Mitral Valve & $2(15 \%)$ \\
\hline \multicolumn{2}{|l|}{ Type of MV Surgery } \\
\hline Mechanical MV Replacement & $6(46 \%)$ \\
\hline Bioprosthetic MV Replacement & $5(38 \%)$ \\
\hline MV Repair & $2(15 \%)$ \\
\hline \multicolumn{2}{|l|}{ Type of Arrhythmia } \\
\hline Paroxysmal AF & $6(46 \%)$ \\
\hline Persistent AF & $6(46 \%)$ \\
\hline MA Flutter & $4(31 \%)$ \\
\hline
\end{tabular}

Table 4: Left atrial endocardial scar data for the 13 patients with pre-ablation sinus rhythm 
voltage maps

\begin{tabular}{|c|c|}
\hline B) Left Atrial Endocardial Scar Data & \\
\hline Average Number of Voltage Points Obtained & $1881 \pm 1293$ \\
\hline Average Area of LA Endocardium (cm $\left.{ }^{2}\right)$ & $124 \pm 46$ \\
\hline Average Area of LA Endocardial Scar (cm ${ }^{2}$ ) & $44 \pm 48.5$ \\
\hline Percent of Surface Area with Endocardial Scar & $29 \pm 23.6$ \\
\hline Scar Burden & \\
\hline Patients with Mild Scar Burden (<29\%) & $8(62 \%)$ \\
\hline Patients with at least Moderate Scar Burden (>29\%) & $5(38 \%)$ \\
\hline Ablation Outcome Data & $\mathrm{N}=8$ \\
\hline Patients with Mild Scar Burden (<29\%) & $5(63 \%)$ \\
\hline Patients Free From Arrhythmia After Index Ablation & $3(37 \%)$ \\
\hline Patients with Recurrence of Arrhythmia & $\mathrm{N}=5$ \\
\hline Patients with at least Moderate Scar Burden (>29\%) & $1(20 \%)$ \\
\hline Patients Free From Arrhythmia After Index Ablation & $3(60 \%)$ \\
\hline Patients with Recurrence & $1(20 \%)$ \\
\hline Patients with Acutely Unsuccessful Ablation Procedure
\end{tabular}

Figure 1: Comparison of baseline sinus rhythm voltage maps in two patients.

A) Baseline sinus rhythm voltage map in a patient with a history of mitral valve repair for severe regurgitation and no prior surgical ablation procedure. This patient had a $16 \%$ scar burden at baseline prior to ablation. This patient had documented paroxysmal atrial fibrillation for which pulmonary vein isolation was performed and had no recurrences at follow up. B) Baseline sinus rhythm voltage map in a patient with a history of mechanical mitral valve replacement for a history of rheumatic valve disease. This patient had a $45 \%$ scar burden at baseline prior to ablation. This patient had documented persistent atrial fibrillation and mitral annular flutter. This patient received pulmonary vein and posterior wall isolation and a mitral annular line 


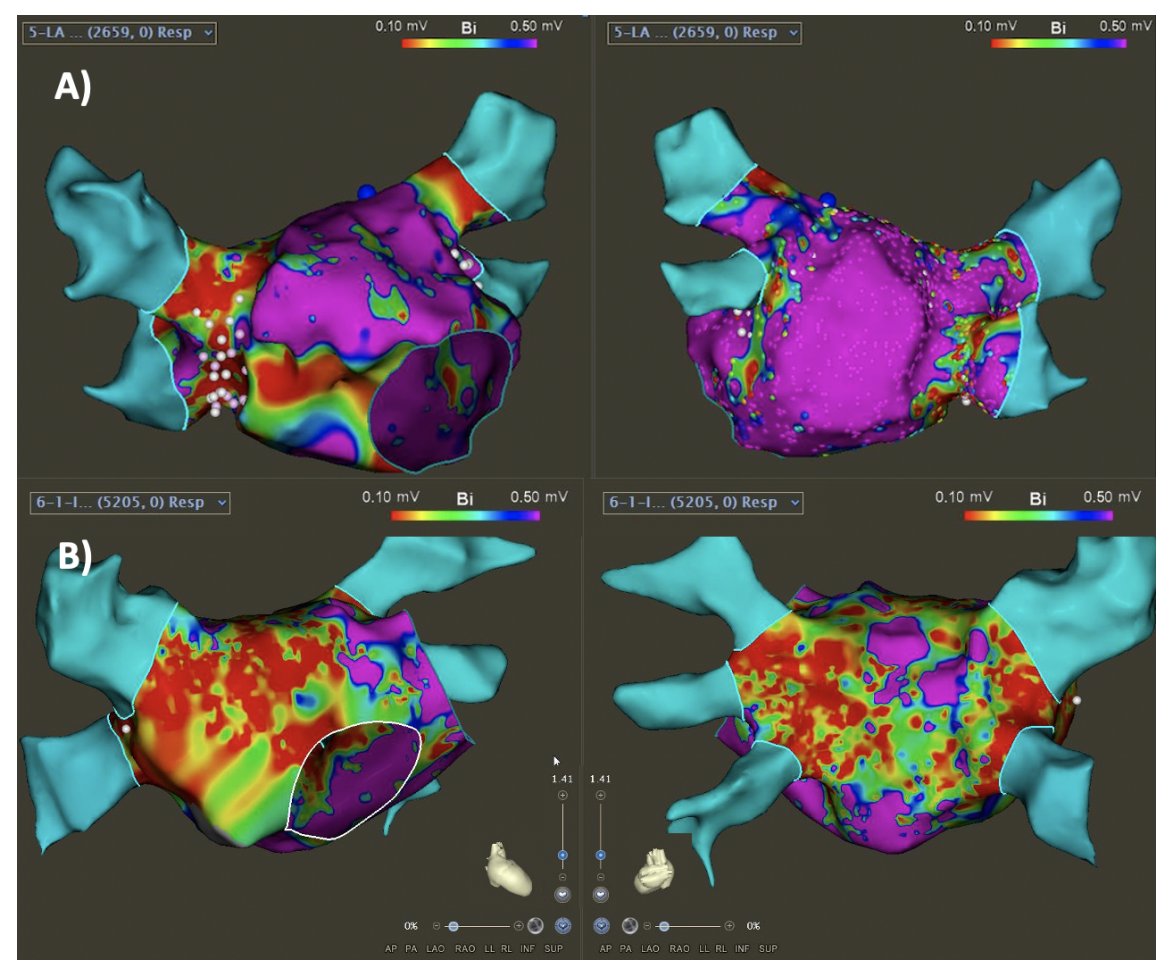

Figure 2: Ablation outcome in relation to burden of left atrial scar

Ablation Outcomes Based on Scar Burden ( $N=13)$

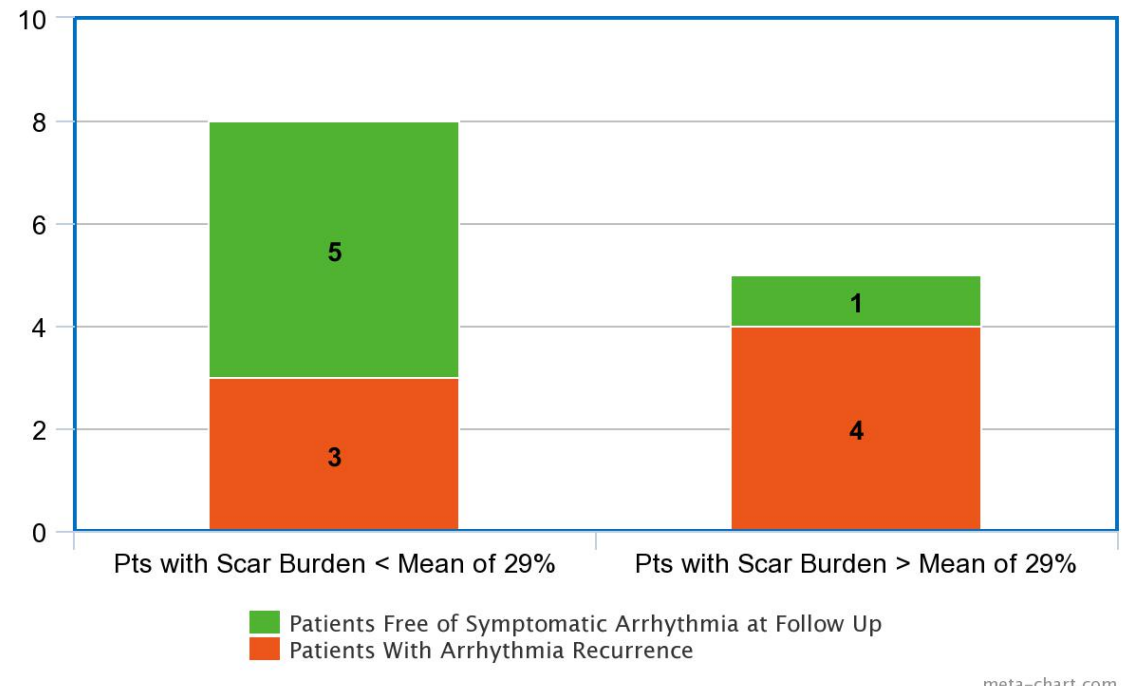

